\title{
EchoGéo
}

16 | 2011

Échos de Turquie

\section{Reconversion(s) territoriale(s) sur l'avenue de Kurtuluş}

Cilia Martin

\section{(2) OpenEdition}

Journals

\section{Édition électronique}

URL : https://journals.openedition.org/echogeo/12393

DOI : 10.4000/echogeo. 12393

ISSN : 1963-1197

Éditeur

Pôle de recherche pour l'organisation et la diffusion de l'information géographique (CNRS UMR 8586)

Référence électronique

Cilia Martin, «Reconversion(s) territoriale(s) sur l'avenue de Kurtuluş », EchoGéo [En ligne], 16 | 2011, mis en ligne le 04 juillet 2011, consulté le 25 août 2021. URL : http://journals.openedition.org/ echogeo/12393; DOI : https://doi.org/10.4000/echogeo.12393

Ce document a été généré automatiquement le 25 août 2021.

EchoGéo est mis à disposition selon les termes de la licence Creative Commons Attribution - Pas d'Utilisation Commerciale - Pas de Modification 4.0 International (CC BY-NC-ND) 


\title{
Reconversion(s) territoriale(s) sur l'avenue de Kurtuluş
}

\author{
Cilia Martin
}

Au cœur de la mégalopole d'Istanbul se trouve l'ancien quartier de Kurtuluş. Construit sur une colline, aujourd'hui à cheval entre la municipalité de Beyoğlu et celle de şişli, Kurtuluş fut d'abord une région isolée du reste de la ville. Anciennement désigné sous le nom de Tatavla ou de Saint Dimitri en référence à son église, Kurtuluş s'est formé à partir du XVI $\mathrm{X}^{\mathrm{e}}$ siècle. Jusqu'à la fin du XIX ${ }^{\mathrm{e}}$ siècle, Tatavla n'est encore qu'un village qui commence, au début du XX' ${ }^{e}$, à s'étirer vers le nord le long d'un axe. Tatavla qui signifie, en grec, les étables, en référence à la période ottomane où la région servait de pâturage aux chevaux, a été rebaptisé Kurtuluş qui signifie, en turc, libération. Cette première emprise territoriale par le biais d'un changement de toponymie a été réalisée après l'avènement de la République turque mais également, selon l'historiographie du quartier et d'après ses habitants, suite à l'incendie de 1929 qui ravagea une partie du quartier. Ce dernier connaît à partir du début du XXe siècle, et à l'instar de la ville, une importante croissance urbaine. Cette dernière est la conséquence d'un exode rural provenant de l'est du pays et relatif à la mécanisation des campagnes. S'ensuit un étalement de l'agglomération urbaine engloutissant Kurtuluş qui devient un quartier péricentral. Outre la transformation du paysage, la ville d'Istanbul connaît également des ruptures démographiques et sociales considérables. Ces dernières se manifestent de manière originale à Kurtuluş car le quartier est un front pionnier minoritaire, dans lequel l'embrassement entre les différentes populations est en partie dû à un chevauchement géographique ${ }^{1}$. Connu comme étant autrefois la "petite Athènes d'Istanbul ", le quartier de Kurtuluş se prolonge au nord-est vers le quartier de Pangalt, ancien bastion de la population levantine (Marmara, 2004), et se confond au nord-ouest avec le quartier de Feriköy qui concentre aujourd'hui la plus grande population arménienne de la ville. Enfin, Kurtuluş est également la troisième destination de migrants provenant de la région d'Erzincan. 
À l'échelle du XXe siècle, l'histoire de Kurtuluş est donc celle d'un quartier en pleine formation puis entièrement recomposé, dont les pratiques territoriales de ses habitants seront analysées à l'échelle d'une avenue.

Pionnière dans la modernisation du quartier, l'avenue de Kurtuluş, héritière de l'avenue de Tatavla, est aujourd'hui l'épine dorsale du quartier. Ancien tracé existant au nord du village de Saint Dimitri, monopole de la communauté rum ${ }^{2}$, l'avenue a été progressivement désertée par elle, au profit d'autres populations. Par ailleurs, l'avenue de Kurtuluş est avant tout une rue marchande dans laquelle les enjeux identitaires et territoriaux passent par le biais du commerce. Espace investi et réinvesti, objet de diverses formes d'appropriation, cette avenue est donc une véritable vitrine de la diversité.

La territorialité est un concept relationnel considéré comme le type de rapport au territoire. Claude Raffestin décrit la territorialité comme «la pratique et la connaissance que les groupes humains mettent en cuvre pour occuper, exploiter et modeler cet espace de manière à le transformer en territoire doué d'une certaine habitabilité » (Raffestin, 1986: 92). Puis, il ajoute que toute théorie sur le territoire est fondée sur l'analyse des relations que les groupes entretiennent avec l'extériorité et l'altérité à l'aide de médiateurs, qui peuvent être la production de signes. Autrement dit, la territorialité est une manière de gérer l'Autre qui se traduit dans l'espace. Par conséquent, le territoire n'est donc pas une zone délimitée, invariable et insignifiante, c'est le produit d'une série, consciente et inconsciente, d'actions humaines qui font l'objet d'une traduction sémiologique.

Par ailleurs, le micro-espace qui nous intéresse ici sera abordé sous le prisme des mobilités en vue de saisir in fine les productions territoriales. En effet, selon Xavier Piolle, "observer le mouvement nous conduit à nous interroger sur les identités et les territoires » (Piolle, 1990 : 153). De plus, Alain Tarrius considère que la mobilité est un fait social qui chamboule les anciennes territorialités tout en créant de nouvelles centralités (Tarrius, 2000). Aussi, le cœur de Tatavla qui se situait autrefois autour de l'église rum de Saint Dimitri s'est-il légèrement décalé vers l'avenue de Kurtuluş, voire s'est désagrégé en plusieurs pôles, autour desquels se concentre une catégorie de population. Par conséquent, ce sont les diverses dynamiques de peuplement qui sont à l'origine de la diversité des espaces vécus et représentés. Ce sont ces flux qui, le temps d'un arrêt, déposent sur le tissu urbain des traces qui, dans leur répétition, deviennent les couches de la sédimentation urbaine. Celle qui fait dire à Alain Corboz que l'espace est un palimpseste (Corboz, 2001) ou à Marcel Roncayolo que «la ville n'est jamais synchrone avec elle-même » (Roncayolo 1990 : 143). Mais qui dit flux et mobilités, sousentend aussi nœuds et ruptures. L'histoire de Kurtuluş et de son avenue est alors une histoire de ruptures. Ces ruptures sont tout d'abord identiques à celles que la ville a connues (départ des minoritaires remplacés par les migrants anatoliens) et par conséquent suivent la même chronologie. Mais, examinées à l'échelle d'une avenue, elles nous servent à dépasser les macro-récits et à cerner, dans toute sa substance, le passage d'une territorialité à une autre voire d'une territorialité unique à des territorialités différentes.

Dans cet article, je propose donc d'interroger les différentes pratiques territoriales qui sont mises en œuvre et qui participent à la recomposition du quartier de Kurtuluş étudiée à travers son avenue phare. Ainsi, j'envisage, tout d'abord, de revenir sur la formation de l'avenue de Kurtuluş, moment qui correspond au passage du village au quartier. Un quartier qui est majoritairement minoritaire, spécifiquement rum, 
jusqu'au milieu du XXe siècle. Le point de départ de la croissance urbaine se situe au sommet de la colline, sur un carrefour entre plusieurs axes, dont celui de Kurtuluş. Ce carrefour, ancienne place publique, possède aujourd'hui de multiples facettes. Si son rôle de centralité sur le plan physique subsiste encore, il ne lui reste plus grand-chose de sa fonction sociale.

Puis, je propose d'étudier le mouvement général de renouvellement de population, plus précisément la passation territoriale entre la communauté rum et les nouveaux habitants à travers la mutation des commerces (changement de propriétaire, relocalisation, renouvellement) et l'apparition d'un "commerce identitaire». Ce qui m'amènera également à parler du changement dans le type de commerce, autrement dit de la juxtaposition des anciens commerces avec les nouveaux commerces franchisés, qui renvoie à différentes pratiques de consommation.

Enfin, depuis les années 2000, alors que le quartier accueille des populations venant de plus en plus loin, il est l'objet d'une nouvelle forme d'appropriation à travers des investissements mémoriels. Plusieurs récits de quartier ont été publiés, notamment dans le cadre d'Istanbul 2010, capitale culturelle européenne. Ils sont le symptôme d'une revitalisation du passé minoritaire et l'expression d'un discours relatif à la mosaïque urbaine érigée en nouvelle valeur patrimoniale et marchande.

\section{La naissance d'une avenue phare, monopole de la communauté rum}

Illustration 1 - Zoom sur une carte datée de 1836 montrant le village de Saint Dimitri et le tracé de la future avenue de Kurtuluş

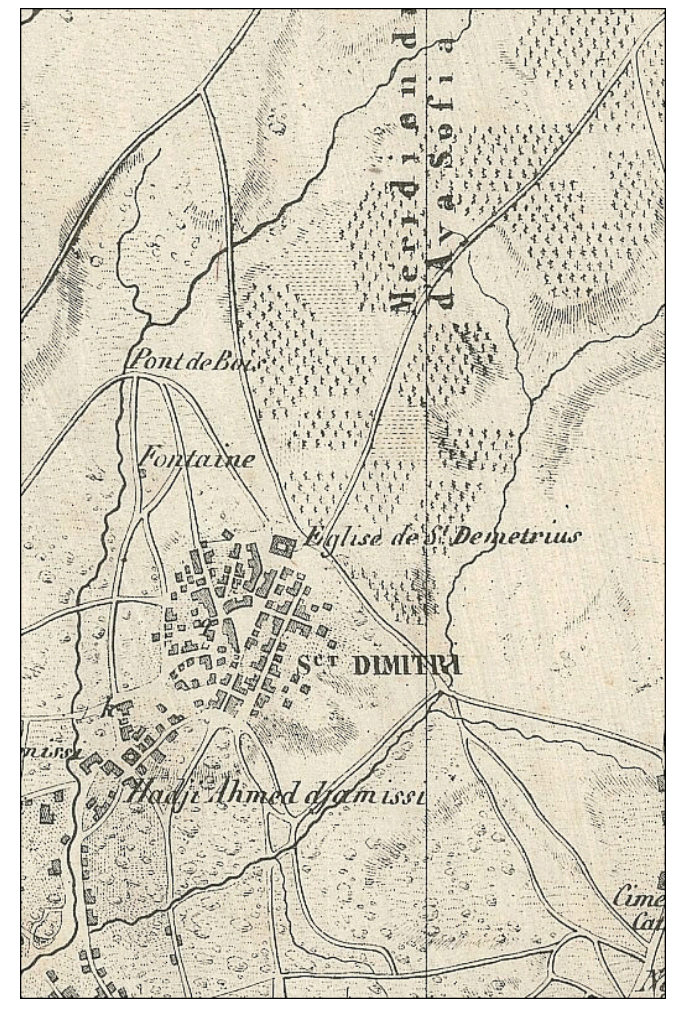

Source : Hellert, 1836 
Illustration 2 - Zoom sur une carte datée de 1866 montrant le début des constructions sur l'avenue de Kurtuluş

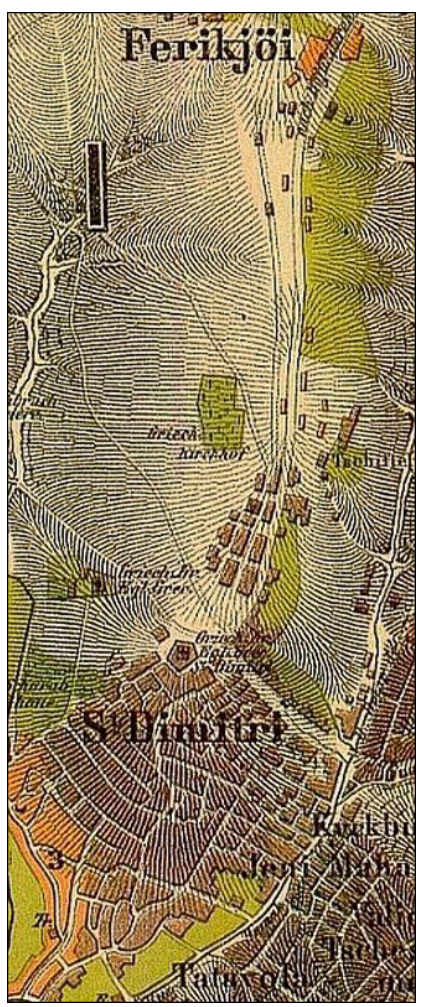

Source : Stolpe, 1866.

$\mathrm{Au}$ début du $\mathrm{XX}^{\mathrm{e}}$ siècle, l'avenue n'existe pas encore. Néanmoins, comme nous pouvons le voir (illustration 1) sur la carte datée de 1836 (Hellert, 1836), l'état du site "porte en germe la structuration du futur tissu » (Panerai, 2005 : 27). En effet, en déroulant le fil des âges, on s'aperçoit que l'avenue de Kurtuluş a été construite sur un tracé, un chemin déjà existant avant l'extension du quartier lui-même. Elle a été édifiée sur un mode de croissance continue, c'est-à-dire dans le prolongement des parties déjà construites. En effet, on aperçoit (voir illustration 2) sur la seconde carte datée de 1866 (Stolpe, 1866), outre le cimetière rum au nord-ouest de l'église rum de Saint Dimitri, quelques constructions à l'orée de l'avenue. Le village de Saint Dimitri qui s'était étendu jusqu'alors sur le versant sud et est de la colline dans une croissance polaire (ordonnée par un pôle, soit l'église de Saint Dimitri), connaît, au début du $\mathrm{XX}^{\mathrm{e}}$ siècle, une croissance linéaire ordonnée par l'axe nord (la future avenue de Kurtuluş). Comme «le passage d'une géographie de pôle à une géographie de ligne signifie la modernisation » (Le Bras, 1998), on passe d'un village centré autour de son église à un nouveau quartier dont le tracé urbain se quadrille en fonction de la répartition des eaux et de l'électricité. Alors que la majeure partie de Kurtuluş est encore dans le noir en 1920 et que seulement $16 \%$ des immeubles sont connectés au réseau électrique, $65 \%$ des immeubles sur l'avenue de Kurtuluş sont éclairés (Sönmez, 2008). Les effets de la modernisation se manifestent également par la ligne de tramwayqui relie, de 1911 à 1966, şişli (terminus de la ligne et, en 1911, de la ville) à Eminonü en passant par Taksim (Kayserilioğlu, 1999). Parallèlement au développement de "l'autoroute» de Tatavla, les immeubles qui longent l'avenue se multiplient. Toutefois, comme nous pouvons le voir ci-dessous (illustration 3), les constructions plus nombreuses en bas de l'avenue (près de l'église) 
se raréfient en allant vers le nord. Les constructions en dur (couleur rouge) et les plus élevées (indication du nombre d'étages) sont majoritaires sur l'avenue alors que les constructions qui s'adossent à la colline sont en bois (couleur jaune). Toutes ces remarques annoncent le caractère prédominant de l'avenue de Kurtuluş par rapport au reste du quartier et nous donnent des indices quant aux différences sociales de ses habitants.

Illustration 3 - Plan cadastral d'assurance relevé par Pervitich en 1925

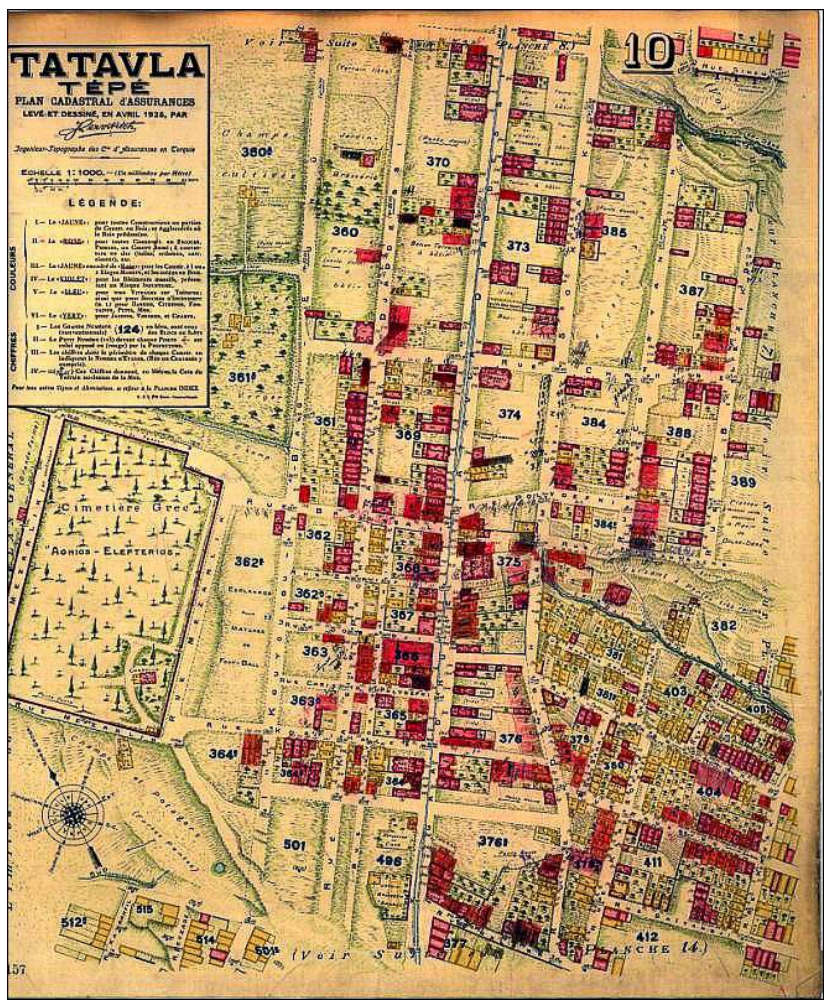

Source : Pervitich, 1925.

Contrairement à une géographie sociale de rivage, courante à Istanbul, qui place la richesse au bord de l'eau et délègue la pauvreté en hauteur, le pied de la colline représente, à Kurtuluş, le départ de l'ascension sociale. Aussi, selon certains anciens habitants rum de Kurtuluş, l'école rum d'Evangelistria, au pied de la colline, accueillait les enfants de familles rum modestes contrairement à l'école au sommet de la colline, mitoyenne de l'église St Dimitri. De plus, la géographie sociale correspond généralement à une géographie confessionnelle. A Kurtuluş, les chrétiens sont au sommet de la colline et les musulmans en contrebas. En effet, le versant sud de la colline de Kurtuluş, en direction de Kasımpaşa, est un ancien quartier musulman, celui de Hacı Ahmet dont son tekke (couvent) date du XVI ${ }^{\mathrm{e}}$ siècle (voir illustration 2, la couleur orange représente les musulmans). Sur le versant est de la colline, se trouve l'actuel quartier de Dolapdere. Encore aujourd'hui, ce dernier, autrement désigné par

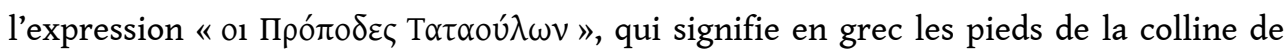
Tatavla, détient dans l'imaginaire de la communauté rum d'Istanbul une place importante, mais pas des plus belles. Réputés pour être un lieu mal famé, les pieds de la colline étaient et sont toujours habités par une population de condition sociale modeste, en grande partie, aujourd'hui constituée de Gitans. Par conséquent, l'avenue 
de Kurtuluş représente à la fois une consécration sociale et une surreprésentation rum, lesquelles sont confirmées par la liste des Rum non-échangeables. Cette liste, conservée aux archives du Ministère des Affaires Etrangères grec, dresse l'inventaire des Rum non inclus dans l'échange de population en 1923 entre la Grèce et la Turquie. Ces Rums qui sont autorisés à rester à Istanbul, sous condition d'y résider avant la date du 31 octobre 1918, ont la nationalité turque et le statut d'Etablis. Ces archives fournissent de précieuses informations démographiques concernant la population rum non échangeable, telles que le nom, prénom et situation maritale, le lieu de naissance, de domicile, d'inscription à l'Etat-civil, la profession et une photographie. Ainsi, selon cette source, sur un ensemble de 174 rues, l'avenue de Kurtuluş représente $12 \%$ de la population totale rum, soit environ 500 Rums sur un total de 4 200. Les rues parallèles qui ont à peu près la même longueur (un kilomètre environ), représentent, elles, à peine $2 \%$ de la population totale. De plus, l'avenue de Kurtuluş présente dans les années 1920 de nombreux terrains à bâtir (voir illustration 3). Autrement dit, même à demi contruite, l'avenue de Kurtuluş est le lieu d'une véritable centralité rum.

Par conséquent, nous avons aujourd'hui deux trames urbaines dans le même quartier, l'une plus récente avec un tracé géométrique qui s'adapte à la modernisation et qui regroupe une population relativement aisée et l'autre plus ancienne au tracé giratoire qui renferme une population de condition sociale plus modeste et entre les deux, une zone floue.

Illustration 4 - Photographie satellite montrant le contraste entre les deux trames urbaines séparées par la place de Kurtuluş

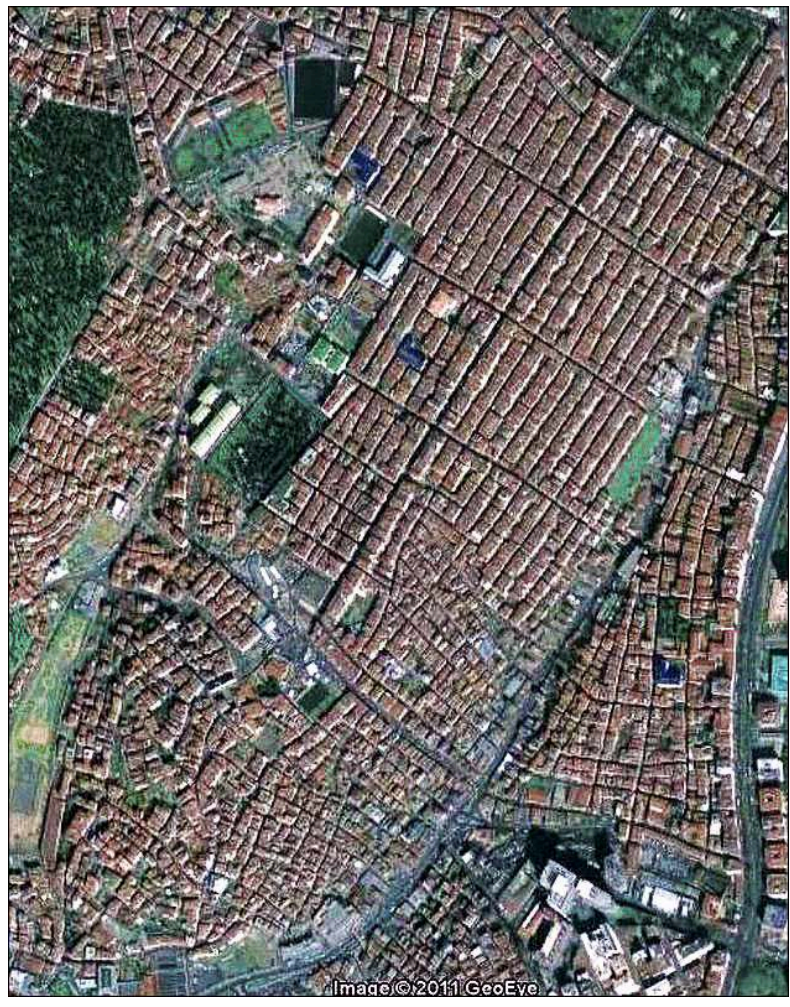

Source : Google earth. 


\section{Objet de lutte territoriale}

Dans la dynamique de croissance urbaine, le centre initial joue souvent le rôle de borne de croissance, en tant que point de départ à une forme de croissance opposée. Il peut s'agir d'un monument, carrefour, pont, gare... Cette borne qui peut aussi être désignée comme une barrière de croissance, introduit, comme nous l'avons vu une coupure dans le tissu mais aussi une "dégradation du tissu au voisinage de la barrière » (Panerai, 2005 : 32). En effet, la place de Kurtuluş offre, de nos jours, un tissu dégradé qui se perçoit également sur les plans de Pervitich. D'une part, la circulation entre les automobiles, autobus, piétons et taxis n'y est pas claire et d'autre part, la place hésite entre être aujourd'hui la place publique qu'elle était autrefois et un terminus pour les autobus. Et, si l'on considère que dénommer, c'est s'approprier, cette place, sans nom officiel mais communément appelée son durak (en turc, terminus), peut-être considérée non pas comme dénuée d'appropriation mais plutôt comme objet de lutte territoriale (Lévy, 2003).

Jusqu'à la veille de la Seconde Guerre mondiale, la place était bien plus vaste qu'aujourd'hui. A titre d'exemple, les habitants du quartier, des quartiers environnants comme des quartiers plus lointains s'y rassemblaient pour célébrer, trois jours durant, le fameux carnaval de Kurtuluş (en turc, baklahorani). Sur la place s'élevaient des manèges pour enfants, une tente de cirque tenu par une famille tzigane et de nombreux music-halls (Burhan, 2003). Les music-halls (en turc, gasino), que l'on retrouvait ailleurs dans la ville, étaient un lieu de divertissement très fréquenté par l'ensemble de la population du quartier et rythmaient sa vie sociale (Irmak, 2003). Rien que sur la place de Kurtuluş, se dressaient trois music-halls. Par conséquent, d'un lieu de sociabilité connu de la ville entière, la place de Kurtuluş est devenue, à partir des années 1970-1980, un endroit en suspens et réduit de moitié. En effet, la place est, de nos jours, bordée au sud par les murs de l'église rum de Saint Dimitri, au nord prolongée par l'avenue de Kurtuluş, à l'est par une station essence et à l'ouest par des cafés et un coiffeur pour hommes. Durant la journée, l'endroit est investi temporairement par des passants et des vendeurs ambulants qui exposent leur étalage de cigarettes, de persil et de tapis. Le soir, à partir de $17 \mathrm{~h}$, la place est un lieu de rassemblement pour la communauté irakienne.

Si la place de Kurtuluş n'a pas vraiment le statut de place publique et ne fédère pas l'ensemble des habitants du quartier, c'est qu'elle est et a toujours été propriété de l'église rum de Saint Dimitri. Tenants du statut de fondation pieuse ou vakıf (Tsistélikis, 2009), les membres du comité d'administration de la communauté rum de Kurtuluş louent une partie des îlots de la place à des supermarchés, restaurants et café. Ces cafés et supermarchés remplacent les anciens music-halls d'hier. A titre d'exemple, le supermarché «Carrefour » qui se trouve aujourd'hui au début de l'avenue de Kurtuluş verse chaque moi un loyer à l'église. Paradoxalement, bien que la communauté rum, du quartier de Kurtuluş et de la ville, soit aujourd'hui démographiquement très réduite, elle est encore présente sur le foncier par l'intermédiaire des vakıfs qui, de fait, gèlent la production territoriale. Malgré ses flottements, la place de Kurtuluş est l'objet d'un récent projet immobilier soutenu par la municipalité de şişli. Ce projet apparaît sur le site Internet de la mairie comme le projet de Kurtuluş Son Durak Projesi (le projet du terminus de Kurtuluş). Ce projet vise à résoudre les problèmes de circulation automobile tout en créant un complexe qui attirerait la population sur la place. Il 
s'intitule Kurtuluş Piazza et vise, comme son nom l'indique, à relancer une idée de place. Une place publique et à vocation internationale si l'on regarde de plus près les terrains d'action de Vizzion Europe, promoteur immobilier responsable du projet. En effet, cette entreprise créée il y 30 ans, par un Turc, est aujourd'hui basée en Belgique et bénéficie d'une structure internationale présente dans cinq pays (France, Luxembourg, Turquie, Serbie et Azerbaïdjan.).

En conclusion, la première emprise territoriale mise en évidence dans cette première partie correspond au moment de formation de l'avenue de Tatavla, rebaptisée avenue de Kurtuluş. Il s'agit d'une appropriation par dynamique de croissance urbaine et de peuplement manifestée par un changement toponymique ${ }^{3}$. En effet, l'avenue de Kurtuluş a été aménagée et investie dans la première moitié du $\mathrm{XX}^{\mathrm{e}}$ siècle, principalement par des Rum voire des Grecs ${ }^{4}$ de condition sociale relativement aisée (contrairement au reste du quartier). La borne de croissance à partir de laquelle s'étend l'avenue est devenue au fil du temps un objet de lutte territoriale (ancienne place publique, propriété de l'église, terminus et projet immobilier) dont découle aujourd'hui une imprécision quant à son statut à la fois spatial et social. La deuxième forme de territorialité dont il sera question concerne le glissement territorial du monopole rum aux nouveaux habitants qui réinvestissent le quartier à partir des années 1950. Date qui correspond aux premiers départs de la minorité rum et aux arrivées massives d'immigrants provenant d'Anatolie, arméniens et musulmans.

\section{Une avenue en transition territoriale}

Les années 1950 représentent un changement dans la balance démographique de la ville et par extension génèrent une profonde transformation urbaine. Dans un chapitre de la thèse d'Ipek Akpınar consacré aux démolitions urbaines à Istanbul dans la décennie 1950-1960, l'auteur désigne sous le terme de Turkification un projet politique nationaliste aux nombreuses répercussions économiques et urbaines (Akpınar, 2004). En effet, l'auteur explique le passage d'une ville ottomane et cosmopolite à une métropole turque par le biais de l'exode rural impulsé par l'industrialisation, des émigrations de non musulmans à l'étranger et par les massives démolitions et reconstructions urbaines. Le milieu du $\mathrm{XX}^{\mathrm{e}}$ siècle est alors un moment clé pour comprendre le processus de recomposition du quartier de Kurtuluş.

\section{Une déterritorialisation rum}

Au début des années 1950, le quartier est largement urbanisé, la population de la ville ainsi que celle du quartier a considérablement augmenté. Selon le recensement général du 22 octobre 1950, sur une population totale de 983041 Stambouliotes, on comptait 42652 Arméniens, 28114 juifs et 66106 Rum. Concernant le quartier de Kurtuluş, on peut se référer aux archives paroissiales de l'église rum de Saint Dimitri dont une liste est issue d'un dénombrement de la communauté rum réaliséen 1960. Les dénombrements étaient effectués par le directeur de l'école rum de Kurtuluş et renouvelés tous les quatre ans à l'occasion des élections communautaires. Seuls les chefs de ménage avec leur prénom, nom et adresse complète figurent sur la liste. La liste fait donc état de 2231 chefs de ménage. Il faut ainsi au minimum multiplier ce chiffre par 4 (une femme et 2 enfants en moyenne) pour arriver à l'estimation suivante 
de 10000 personnes. Un membre du comité d'administration de la communauté rum de Kurtuluş depuis 1960, qui était là au moment du dénombrement, m'affirme que la communauté rum de Kurtuluş s'élevait alors à 11000 personnes, soit $15 \%$ de la population rum totale. L'on considère aujourd'hui que la moyenne générale d'un mahalle à Kurtuluş, selon les chiffres donnés par la municipalité de şişli, s'élève à 13000 personnes. On peut estimer que dans les années 1960, le mahalle en compte environ la moitié, c'est-à-dire 6000 personnes. Toujours par hypothèse, Kurtuluş qui s'étend sur 5 mahalles aurait donc eu, dans les années 1960, une population approximative de 30000 personnes, dont 10000 Rums, soit $33 \%$. Indéniablement, Kurtuluş est encore, en 1960, un quartier à forte composante rum. Parmi cette population rum, il faut compter les Rum originaires de Kurtuluş, ceux venus d'autres quartiers de la ville et les Grecs revenus après l'accord de 1930. Le reste de la population se compose d'Arméniens venus majoritairement de l'Est de la Turquie, de juifs venus le plus fréquemment d'Ortaköy, de Balat ou de Galata, ou de musulmans venus d'autres quartiers de la ville ou d'ailleurs en Turquie. Dans la moitié du XXe siècle, le quartier de Kurtuluş, et plus généralement celui de şişli, devient donc un quartier à la mode (on parle de la femme de şişli pour désigner un critère de beauté), un quartier attractif pour une population enrichie qui décide de quitter la péninsule historique pour vivre dans un quartier qui se veut plus moderne.

Néanmoins, les évènements du 6 et 7 septembre 1955 marquent une rupture. Dans la nuit du 6 au 7 septembre 1955, suite à des tensions à Chypre et à l'annonce d'un attentat commis à Thessalonique dans la maison où Atatürk est né, des pillages et violences ont été commis à l'encontre des chrétiens de la ville dont les Rum qui ont été la cible majoritaire (Akar, Demir, 1994). Sur l'avenue de Kurtuluş, et dans d'autres rues du quartier, une partie des commerces tenus et des maisons occupées par des rum ont été également saccagés. A partir de cette date, la reconversion territoriale s'opère par une redistribution des présences et par extension de la visibilité. Cette reconfiguration s'amplifie en 1964 avec le départ des ressortissants grecs. Les accords de 1930 entre Vénizelos et Atatürk n'étant pas été reconduits, ils sont contraints de quitter la Turquie et sont suivis par un grand nombre de Rum avec lesquels ils se sont mariés.

1955 et 1964 sont deux dates clés qui, par conséquent, se traduisent aussi bien par les départs des minoritaires rum que par leurs délocalisations et relocalisations à l'intérieur du quartier. En effet, sur une seconde liste issue des archives paroissiales de Kurtuluş et datée de 1964, des informations ont été rajoutées pour signaler les mobilités des habitants sous la forme de déménagement, départ, ou décès. Parmi 965 déplacements, outre les rares décès, on compte 453 départs en Grèce et ailleurs ( soit $47 \%$ ), 96 déménagements à l'intérieur de la ville (soit $10 \%$ ) et 383 déménagements au sein même du quartier (soit $40 \%$ ). De plus, si l'on compare l'ancienne population (présente sur la liste de 1960 et sur celle de 1964) avec la nouvelle population (absente sur la liste de 1960 et présente sur celle de 1964), on remarque que la nouvelle population se déplace presque deux fois plus que l'ancienne (688 déplacements chez la nouvelle population contre 418 chez l'ancienne). Toutefois, quand l'ancienne population bouge, elle se déplace à $70 \%$ dans le quartier alors c'est le cas pour seulement $35 \%$ de la nouvelle population. Ces chiffres nous apprennent, d'une part, que l'attachement au territoire, traduit 'par un sentiment d'inscription spatiale' (GuérinPace France, 2004) est plus fort chez l'ancienne population que chez la nouvelle pour laquelle on enregistre plus de départs. D'autre part, on constate, de manière générale, 
que la réaction des habitants rum face aux événements politiques, qui les menacent de près ou de loin, est la mise en mouvement. Un mouvement qui prend la forme d'un départ vers la Grèce mais également d'un nouvel ancrage dans le quartier, voire dans la même rue. Enfin, si l'on compare la liste de 1960 avec la dernière liste issue du dénombrement de 2005, on constate que les anciens habitants rum (ceux qui sont présents depuis 1960) se sont retirés du devant de la scène. En effet, ils n'habitent pas ou plus sur l'avenue de Kurtuluş. Ce dénombrement réalisé par un démographe grec, en 2005, à l'occasion d'un colloque à Istanbul ${ }^{5}$, fait état de 800 Rum à Kurtuluş contre 10000 dans les années 1960. Ce déclin démographique causé par les événements de 1955, 1964 et 1974 (suite aux évènements de Chypre) se double d'un effacement spatial illustré par une nouvelle géographie sociale. La communauté rum déserte aujourd'hui la colline pour se cristalliser au sommet, notamment autour de l'église rum de Saint Dimitri, ancien cœur de Tatavla. Par ailleurs, leur effacement sur le plan spatial se manifeste également par un manque de mobilité et de lien social. En effet, la plupart des anciens habitants rum sont aujourd'hui très âgés et isolés, une partie de leur famille se trouvant en Grèce ou à l'étranger. Cet enclavement à la fois spatial et social est à l'origine d'une déterritorialisation (Haesbaert Da Costa Rogério, 2004). Si le quartier est par conséquent, pour les uns, synonyme de déterritorialisation, il est pour d'autres, l'objet d'une nouvelle appropriation.

\section{Une territorialité plurielle}

Les nouvelles appropriations qui se manifestent sur l'avenue de Kurtuluş seront appréhendées par le biais de la mutation des commerces. Celle-ci se décline de trois façons : la première coïncide avec un changement de propriétaires; la deuxième est incarnée par l'arrivée de nouveaux commerçants qui s'approprient l'avenue tout en marchandant leur identité; la troisième correspond à une relocalisation et un renouvellement spatial des commerces et à l'arrivée de nouveaux types de commerces.

Premièrement, le changement de propriétaire (en turc, el değiștirmek) est très fréquent au milieu du XX $X^{e}$ siècle. Le sens dans lequel le changement s'effectue est un révélateur des relations inter-communautaires au sein du quartier et de la ville. Ainsi, prenons pour exemple, le passage de Feriköy au bout de l'avenue de Kurtuluş. Anciennement baptisé passage de l'Atlas et dont le propriétaire était rum, le passage était, en grande partie, consacré au commerce de la chaussure. Suite aux événements de 1955, le propriétaire quitte le quartier. Avant de partir, il vend les magasins du passage à des Arméniens, également propriétaires de magasins de chaussures. C'est le cas d'un Arménien né à Kayseri en 1928 et arrivé à Istanbul, alors âgé de deux mois. Il réside d'abord à Taksim puis à Eminönü où il possède un magasin de chaussures. Au cours de la nuit du 6 au 7 septembre 1955, son magasin est détruit. Son propriétaire décide de s'éloigner du centre de la ville et quitte Eminönü pour emménager à Kurtuluş où il achète un magasin de chaussures au propriétaire du passage. Son fils en est aujourd'hui le propriétaire. Par conséquent, le changement de propriété qui s'effectue en grande partie dans le sens rum $\rightarrow$ arménien est un indicateur de la proximité entre ces deux communautés. Encore aujourd'hui, cette tradition de proximité est observable au sein des deux populations par le biais des mariages mixtes. La communauté arménienne s'est donc faite le relais de la communauté rum. Kurtuluş est aujourd'hui connu pour être un quartier à très forte composante arménienne (Hofmann, 2002). Son centre de gravité se situe toutefois plus au nord et, chronologiquement il est plus récent que celui 
de la communauté rum. En effet, c'est à Feriköy (voir illustration 2) que se trouvent une église arménienne construite en 1932, un cimetière arménien et une école arménienne. Par conséquent, l'incertaine frontière spatiale entre les deux quartiers fait écho à la frontière communautaire entre Arméniens et Rum.

Dès la seconde moitié $\mathrm{du} \mathrm{XX}^{\mathrm{e}}$ siècle, l'avenue compte également de nombreux musulmans originaires d'Anatolie qui investissent le quartier. L'avenue met en scène cette nouvelle diversité au moyen des enseignes de commerces qui illustrent une affirmation identitaire et une volonté d'ancrage territorial. En effet, certaines enseignes affichent l'origine géographique de leur premier propriétaire, voire la date de création du commerce. A titre d'exemple, on trouve onze enseignes qui mettent en avant l'origine géographique. De plus, l'affirmation identitaire participe de l'image de marque de certains produits tels que les baklavas de Gaziantep, ville que l'on retrouve écrite sur quatre enseignes différentes. Comme le dit Emmanuel Ma Mung, «dans les transactions marchandes mettant en cause des partenaires spécifiés par leur appartenance à des groupes, se négocient, par le truchement des biens matériels échangés, des valeurs et des signes d'appartenance » (Ma Mung Emmanuel, 2006 : 83). Par le biais de l'échange commercial, s'effectue donc une négociation identitaire voire un marchandage identitaire. Toujours dans le même ordre d'idée, l'implantation d'un bakkal (épicier)est pour les familles originaires, par exemple de Kastamonu, Erzincan, Sivas ou encore Çankırı, le premier point d'ancrage dans le quartier. Lieu de sociabilité important, le bakkal a ainsi légitimé leur présence et sert de repère à toute une catégorie de population. De même, les associations de pays (hemşehri) participent à ce marquage territorial. La première qui date de 1952 et représente les immigrés d'Erzincan, n'est pas présente sur l'avenue mais, comme toutes les autres associations, est reléguée au second plan, dans les rues adjacentes. Cette association de pays qui succède à un ancien music-hall est propriété de l'église rum.

La reconversion territoriale s'effectue enfin par une relocalisation des commerces et plus récemment par l'arrivée de nouveaux magasins franchisés et de nouvelles banques qui ponctuent l'avenue. Tout d'abord, la relocalisation des commerces est illustrée par l'exemple d'une pâtisserie tenue par un Rum dans les années 1940 qui, selon son propriétaire actuel, se trouvait à l'origine de l'autre côté de l'avenue trois numéros plus haut. Dans les années 1960, l'immeuble fut rasé et le propriétaire délocalisa sa boutique trois numéros plus bas. Au changement de propriétaire, le nouvel acquéreur déplaça la boutique là où elle se trouve aujourd'hui. Autre exemple de saut de puce, un bakkal, créé il y a 35 ans par un commerçant originaire de Çankırı, a été déplacé 100 mètres plus haut au moment des travaux et du changement de propriétaire, du père au fils. Outre la relocalisation, le renouvellement des commerces sur l'avenue de Kurtuluş, est relativement rapide. En effet, l'espérance de vie moyenne d'un commerce, depuis les années 1953, est de 11 ans. Cette moyenne a été calculée, pour la période de 1953 à 2010, d'après les registres de la Chambre de Commerce à Istanbul qui indiquent la date d'ouverture et de fermeture des commerces. Si le haut de la hiérarchie n'est, aujourd'hui, plus représenté par les Rums qui ne tiennent aucun commerce sur l'avenue, il revient aux Arméniens. Ainsi, à l'instar de plusieurs magasins de chaussures dans le passage de Feriköy, d'un vendeur de journaux et d'un magasin de textile pour maisons qui date de 1959, les plus anciennes boutiques sont tenues par des Arméniens. Cependant, toujours d'après l'étude des registres de commerce d'Istanbul, sur 268 commerces enregistrés, la proportion la plus grande de commerces a une durée de vie 
de 4 ans. Ainsi, les mobilités commerciales qui sont aujourd'hui inhérentes à l'avenue commerçante de Kurtuluş participent pleinement à sa recomposition.

Enfin, parallèlement à ces quelques exemples d'anciens commerces, de nouveaux types de commerces apparaissent depuis les années 1980. Ce sont principalement des magasins franchisés dans le secteur alimentaire. Aussi, l'avenue de Kurtuluş totalise neuf supermarchés dont un "Carrefour» près de la place centrale. Quatre grands magasins représentent le secteur de l'électroménager. Quasiment toutes les banques de Turquie ont une succursale sur l'avenue. Enfin, parmi ces commerces mondialisés, on remarque des magasins symbolisant une mobilité d'échelle internationale. C'est le cas des call shop dont le premier est apparu sur l'avenue en 2009. Ces derniers sont devenus un lieu de rendez-vous devant lesquels se regroupent les Africains qui stationnent temporairement à Istanbul dans l'attente de migrer vers l'Europe.

Le commerce semble être, par conséquent, un prisme approprié pour saisir la reconversion territoriale sur l'avenue de Kurtuluş. Ainsi, la disparition de l'ancienne place publique de Kurtuluş, la déterritorialisation de la communauté rum au profit de la communauté arménienne et a contrario la territorialisation de nouveaux habitants a produit de multiples polarités symbolisées par l'église, le bakkal, le call shop oul'association de pays. Cette reconversion a pris depuis une dizaine d'années un nouveau tournant. Elle joue cette fois-ci sur le champ de la mémoire comme nouveau moyen d'appropriation du territoire. Les productions mémorielles et les politiques de patrimonialisation contribuent à réactiver le caractère minoritaire du quartier et à diffuser le mythe de la mosaïque urbaine comme valeur promotionnelle et marchande.

\section{Une avenue à la mémoire de la communauté rum}

Depuis une vingtaine d'années, la ville est l'objet d'une importante production mémorielle sous la forme de publications de récits de quartier. Si les anciens quartiers de Kuzguncuk, Üsküdar, Kadıköy ou encore Beyoğlu ont été les premiers concernés, dernièrement, et avec le programme Istanbul 2010, capitale culturelle européenne, des quartiers plus éloignés comme Maltepe, Çengelköy, Göztepe ou encore Ataköy, sont intégrés dans la dynamique d'une production plus localisée de la mémoire urbaine. Dans une dynamique de promotion internationale de la ville, ils contribuent ainsi à redessiner les nouvelles frontières d'Istanbul et à justifier son image de métropole ${ }^{6}$. Concernant le quartier de Kurtuluş, trois récits de quartier ont vu le jour depuis 2002. Le premier est celui de Yorgo Valasiadis, intitulé kai sta tatavla gioni (il neige sur Tatavla) publié en 2002 à Athènes. Puis, il s'agit du récit d'Hüseyin Irmak, Istanbul'da bir kadim semt, yaşadığım Kurtuluş (Un vieux semt à Istanbul, le Kurtuluş où j'ai vécu) publié en 2003. En 2005, sort la version turque du récit de Yorgo Valasiadis. Enfin, le dernier est Feriköy anılarda...şimdi (Des souvenirs...maintenant) écrit par Talin Büyükkürkciyan. Son livre est paru en janvier 2010 au sein du projet My Istanbul. Dans ce récit de quartier sur Feriköy, on trouve quelques passages sur Kurtuluş puisque que l'auteur y a vécu et qu'elle considère qu'il est impossible de parler de Feriköy sans parler de Kurtuluş.

Parallèlement à ces récits qui sont de véritables travaux de mémoire, il existe d'autres ouvrages que je définirai comme des investigations historiques. Ce sont les livres d'Orhan Türker, Osmanlı Istanbulu'ndan bir köşe Tatavla (Tatavla, un coin de l'Istanbul ottoman) paru en 1998 et de Burhan Yentürk, Ne lazım Tatavla'da bakkal dükkanı (Il y a 
tout ce qu'il faut à Tatavla) sorti en 2001 et qui se veulent scientifiques et non mémoriels.

La structure des récits de quartier est quasiment la même dans tous les livres. L'auteur débute par des anecdotes concernant ses ancêtres, leur arrivée dans le quartier, puis poursuit par la description de sa maison, de ses voisins de paliers et d'immeubles. Puis, l'auteur attache une importance particulière à la rue, aux différents immeubles et aux magasins. Evidemment, de nombreux passages sont dédiés à l'avenue de Kurtuluş, à ses épiceries, ses pâtisseries, boucheries, boulangeries... Hüseyin Irmak décrit dans son récit des pans de rues entiers, immeuble par immeuble, numéro par numéro en y donnant le nom de la famille qui y habitait, le nom de chaque membre, leur description physique, leur caractère, leur profession... De plus, les lieux de sociabilité occupent une place prédominante dans le récit. Aussi, on retrouve immanquablement les music-halls, les tavernes, les cinémas, les écoles, les épiceries, les tramways et autobus... Enfin, concernant le style de l'écriture, le champ lexical est celui d'une grande nostalgie. « $A$ cette période, Kurtuluş c'était complètement autre chose » (Irmak, $2003: 35$ ), "Après rien ne fut comme avant»(Irmak, 2003: 43), ou encore "C'étaient des années inoubliables à jamais » (Valasiadis, $2005: 21$ ).

Revenons un instant sur le profil sociologique des auteurs. Yorgo Valasiadis est un ancien habitant rum du quartier qui vit aujourd'hui en Allemagne. Talin Büyükkürkciyan est une turque d'origine arménienne qui vit aujourd'hui à Beyoğlu et enseigne à l'université de Bilgi au département d'art et performance. Enfin, Hüseyin Irmak est kurde, arrivé dans le quartier en 1965. Hüseyin Irmak est la figure pionnière de la réactivation du passé rum de Kurtuluş.

Hüseyin Irmak est né en 1961 à Zara, près de Sivas. Il est arrivé à Kurtuluş à l'âge de 4 ans alors qu'il ne connaissait que la langue kurde. Hüseyin habite aujourd'hui à Kağıthane où il travaille à la mairie comme conseiller à l'édition. Dans son livre, il décrit un quartier où l'on sait côtoyer et bien vivre avec les différences des uns et des autres. Une harmonie parfaite exprimée à plusieurs reprises: "Les enfants Rum, Arméniens, Turcs, Kurdes, tous ensemble nous regardions avec intérêt »(Irmak, $2003: 49)$, «Si Ahmet, Hasan, Hüseyin était naturel pour nous, Agop, Niko, Yasef l'était tout autant » (Irmak, 2003: 123). Des différences qui s'effacent même à l'intérieur de la communauté musulmane puisqu'il écrit «Dans nos maisons, Alevis, Sunnites, tous ensemble nous mangions »(Irmak, 2003 : 27).Ainsi, pour Hüseyin Irmak, c'est l'appartenance au quartier qui définit l'identité et gomme les différences. En 2011, son récit de quartier va être édité pour la troisième fois. Son auteur envisage également de créer un site Internet et de monter une association.

Par conséquent, le profil des auteurs nous rappelle que, paradoxalement, ce sont souvent les personnes extérieures, non Rum ou vivant hors du quartier, autrement dit ceux qui ne sont pas directement dépositaires de la mémoire rum du quartier, qui en deviennent les fervents promoteurs. Hüseyin Irmak me dira lui-même lors d'une entrevue «Istanbul, rum demek » (Istanbul veut dire rum).

Si l'on se place dans une rhétorique du premier occupant (Copeaux, 1998 : 29), les Rum sont les premiers arrivés ou anciens habitants ilk gelen et sont suivis par les nouveaux habitants "sonra'dan gelen ». Les seconds en se revendiquant défenseurs de la mémoire rum, s'identifient aux premiers ilk gelen et ce faisant, sont dans un processus d'appropriation du quartier. Ici, le processus d'appropriation passe par une identification à l'Autre. Les pratiquants de ce discours se placent alors dans une 
certaine continuité par rapport au passé rum et par extension sont généralement dans un rejet des nouveaux habitants, représentés par l'image de l'immigrant originaire d'Erzincan qui n'a jamais su intégrer les valeurs de la ville alors que, par opposition, le rum était bien habillé et bien éduqué. Selon Hüseyin Irmak, « aujourd'hui la plupart des Stambouliotes viennent d'ailleurs, et ne se sentent pas concernés par l'histoire de leur quartier. Ils n'ont que leur village en tête». C'est la raison pour laquelle il milite pour la conservation du passé rum par le biais d'une initiative patrimoniale qui participe à la réification d'une certaine image rum de Tatavla: la reconstitution du carnaval de Kurtuluş.

\section{Tatavla, une nouvelle valeur patrimoniale et marchande}

Depuis trois ans, Hüseyin Irmak organise chaque année, au mois de février, le carnaval de Kurtuluş. L'an dernier, en 2010, ils étaient environ 200 personnes à défiler, costumés, dans les rues du quartier contre une cinquantaine de personnes la première année. Selon Hüseyin, d'anciens habitants rum ont assisté au défilé depuis leur balcon, les larmes aux yeux. En 2011, on parle de 400 participants. Toutefois, malgré l'enthousiasme des organisateurs qui sont le président du conseil d'administration rum de Kurtuluş, quelques étudiants grecs et Hüseyin Irmak, le succès du carnaval reste mitigé. D'une part, un grand nombre de commerçants et de personnes qui ont vu le défilé m'ont dit n'avoir pas compris de quoi il s'agissait. Le reste des habitants n'était simplement pas au courant de ce qui s'était passé comme le conseiller du maire que j'ai rencontré trois semaines plus tard. D'autre part, sur les 300 personnes qui ont assisté à la soirée organisée, seulement une cinquantaine d'habitants du quartier était présente. Le prix de la soirée fixé à 25 lires, coût relativement élevé pour les habitants du quartier, a dissuadé les quelques participants qui ont défilé dans les rues à poursuivre la soirée. Cette dernière s'est déroulée dans un lieu symbolique de la communauté rum, son club sportif. Au cours de la soirée, trois groupes de musique rebétiko, à l'origine la musique des échangés d'Istanbul et de Smyrne, sont venus jouer sur scène à tour de rôle. On dansait et trinquait à Tatavla. La plupart des participants étaient soit des étudiants étrangers amis des organisateurs et des musiciens, soit des touristes, soit d'anciens rum vivant aujourd'hui à Athènes, soit des journalistes.

Par conséquent, cette initiative patrimoniale, non relayée par les pouvoirs publics, a été délocalisée si l'on considère que la plupart des habitants du quartier n'a pas été mise au courant de l'évènement. Cette initiative patrimoniale d'ordre personnel et spontané provient d'une petite poignée d'individus qui agit dans un cercle restreint de personnes gravitant, de plus ou moins près, autour de la communauté rum. Les raisons qui conduisent les organisateurs à reconstituer le carnaval sont spécifiques à chacun. Pour les uns, les membres du conseil d'administration, il s'agit de dynamiser la communauté. Pour les musiciens, cela fait partie du travail, il s'agit de se faire entendre et connaître du public. Quant aux étudiants, outre les questions de prestige, ils participent à un phénomène plus général qui consiste à réévaluer «les relations entre la tradition et la convivialité " (Seraïdari, 2010). Une convivialité qui, bien qu'ayant eu un faible retentissement chez la population locale, a connu un relatif succès grâce à l'effet Facebook ${ }^{7}$ et à son réseau social via lequel la plupart des participants ont été invités. De plus, le carnaval a été extrêmement apprécié et commenté sur Facebook alors que la presse turque n'a que très peu couvert l'évènement. 
Outre les récits de mémoire et le carnaval, il existe quelques personnes qui essaient de profiter de cette instrumentalisation de l'image populaire et minoritaire de Tatavla. Ainsi, parmi les nombreux music-halls et tavernes que l'on trouvait dans le quartier, il reste une taverne ou du moins son nom, Madam Despina. Cette dernière, devenue véritable légende locale, était la propriétaire d'un café. Le propriétaire actuel du café, qui est aujourd'hui un restaurant (Despina Meyhanesi), est un Turc qui travaille à la mairie métropolitaine d'Istanbul. Il vit à Kurtuluş depuis quarante ans. Il me dit avoir parlé grec étant petit. Il prône l'amitié gréco-turque qu'il veut faire revivre dans son magasin. La décoration du restaurant entretient une ambiance rum avec un portrait de Despina sur le mur ainsi que des anciennes photographies. Le soir, des musiciens viennent jouer de la musique tzigane ainsi que de la musique rebétiko. Toutefois, le propriétaire a un discours très négatif concernant les immigrés d'Erzincan vivant en grand nombre dans le quartier.

Par ailleurs, la revitalisation de l'image minoritaire de Tatavla, est observable sur la place de Kurtuluş où l'on remarque un restaurant baptisé Tatavla dürüm evi (La maison des dürüm (sandwich turc) de Tatavla). Enfin, profitant de ce que la musique grecque soit à la mode ces dernières années à Istanbul, un groupe de musiciens, composé de Grecs et Turcs, joue chaque semaine un répertoire rébétiko sous le nom de Tatavla Keyfi (keyif se traduit par le plaisir, la bonne humeur). Ce même groupe a joué le soir du carnaval.

Ainsi, la folklorisation des anciennes pratiques de Kurtuluş fait écho à un réinvestissement patrimonial que l'on observe dans les anciens quartiers d'Istanbul, voire dans d'autres pays. En effet, la tendance générale à Istanbul est au culte de l'ancien. Dans une ville qui veut jouer un rôle sur la scène internationale (Istanbul a été promue capitale européenne de la culture), les anciens quartiers minoritaires redeviennent, pour certains groupes sociaux, attractifs (Islam, Behar, 2003). Dans ces quartiers, la mosaïque culturelle d'Istanbul devient une pièce clé du marketing urbain. Ainsi, on peut lire sur le site Internet de la municipalité de şişli qu' « une attention spéciale est accomplie dans le but d'enrichir la mosaïque culturelle » et plus loin «jusqu'à la république turque, Tatavla était le lieu calme où vivait une grande majorité de Rum, d'Arméniens et une petite quantité de Juifs. Après être devenu Kurtuluş, cette spécificité a duré un certain temps mais dans les années 1990, Kurtuluş a été victime d'érosion». La municipalité, dirigée par un maire originaire d'Erzincan, utilise par conséquent le même discours nostalgique fondé sur un cosmopolitisme disparu. Toutefois, la mairie de şişli n'a pas de véritable politique patrimoniale en ce qui concerne le quartier. Dans une municipalité aussi importante que celle de şişli, le passé rum de Kurtuluş n'attire pas l'attention des pouvoirs publics qui se concentrent sur son électorat originaire d'Erzincan.

Enfin, l'argument de la mosaïque urbaine devient une valeur marchande pour certains promoteurs immobiliers. En effet, sur la brochure du projet Elysium Cool-Kurtuluş de l'entreprise immobilière OFTON, l'argument de vente est le suivant: Kurtuluş est devenu un centre pour le bien vivre ensemble, une mosaïque religieuse où il fait bon habiter.

Par conséquent, la dernière forme de territorialité, la plus récente chronologiquement, prend la forme de stratégies mémorielles qui visent l'appropriation du quartier par le biais d'une identification à l'Autre. Elle s'inscrit dans un phénomène plus général, celui de la reconquête des quartiers historiques d'Istanbul voire de gentrification dans lequel 
Kurtuluş ne se résume plus qu'à Tatavla. Dans ce cadre, les minoritaires, aujourd'hui invisibles sur la scène stambouliote, sont symboliquement mis sur la sellette par une certaine catégorie de la population, la même qui fait le constat d'une ville vidée de ses véritables habitants et envahie par les gens de l'est.

A l'échelle du $\mathrm{XX}^{\mathrm{e}}$ siècle, l'avenue de Kurtuluş s'est entièrement urbanisée et recomposée. Cette recomposition est en grande partie le fruit des mobilités qui redessinent le contenu, à la fois social et spatial, de la ville et de ses quartiers. Des mobilités qui s'illustrent par le renouvellement de la population, par les transformations urbaines et la mutation des commerces, le tout donnant naissance alors à une diversité de pratiques territoriales qui renvoient aux différentes manières de gérer son rapport au voisin (identification, catégorisation, stigmatisation...). L'avenue de Kurtuluş est ainsi passée d'une déterritorialisation rum à une mutliterritorialité dans laquelle se juxtaposent aujourd'hui différentes centralités débouchant sur un lieu confus, la place de Kurtuluş. Si l'avenue de Kurtuluş présente un intérêt aussi important, c'est parce qu'elle est tout d'abord prise dans une dynamique d'étalement urbain, parce qu'elle est le témoin de diverses formes d'appropriation et enfin parce qu'elle est au coeur de nouvelles stratégies mémorielles. Toutefois, c'est aussi du côté de sa contradiction avec le reste du quartier qu'il faudra aussi se tourner.

\section{BIBLIOGRAPHIE}

Akar R., Demir H., 1994. Istanbul'un Son Sürgünleri. Istanbul, İletişim, 230 p.

Akpinar I., 2003. The rebuilding of Istanbul after the plan of Henri Prost 1937-1960: from secularisation to Turkish Modernisation. Thèse de doctorat, University of London, $294 \mathrm{p}$.

Behar D., Islam Tolga, 2006. İstanbul'da "soylulaştırma". İstanbul Bilgi Üniversitesi Yayınları, 256 p. Burhan Y., 2003. Tatavla Panayırı. In Burhan Y., Ne lazım Tatavla'da bakkal dükkanı, Ankara, Geyik Yayınlar1, 2003, p. 38-45.

Copeaux E., 1998. Espace et temps de la nation turque, analyse d'une historiographie nationaliste (1931-1993). Paris, CNRS Editions, Collection Méditerranée, 369 p.

Corboz A., 2001. Le territoire comme palimpseste et autres essais. Paris, Ed. de l'Imprimeur, Collection tranches de villes, $281 \mathrm{p}$.

Guérin-Pace F., 2007. Le quartier entre appartenance et attachement : une échelle identitaire ? In Authier J-Y., Bacqué M.-H., Guérin-Pace F., Le quartier, Enjeux scientifiques, actions politiques et pratiques sociales, Paris, La Découverte, 2007, p. 151-162.

Haesbaert Da Costa R, 2004. De la déterritorialisation à la multiterritorialité. In Allemand S., Ascher F., Lévy J., Les sens du mouvement, Paris, Belin, p. 69-79.

Hofman T., 2002. Armenians in Turkey Today, A critical Assessment of the situation of the armenian minority in the turkish republic. The EU Office of Armenian Associations of Europe, Bruxelles, $45 \mathrm{p}$. 
Irmak H, 2003. Istanbul'da bir kadim semt, yaşadığım Kurtuluş. Istanbul, Aras, Tanıklık, 158 p.

Kanserilioğlu S R, 1999. Dersaadet'ten İstanbul'a tramway II. İstanbul, İ.E.T.T, 274 p.

Kauffer, Le Chevalier, Barbie du Bocage, De Hammer J., 1836. Plan de Constantinople et de ses faubourgs avec l'indication des quartiers de la Ville (Mahallé) et les changements qui y sont opérés depuis les années 1776 et 1786 jusqu'à l'année 1831 par J. Helbert. Paris, cartothèque de l'IFEA, Istanbul.

Le Bras H, 1993. La planète au village. Migration et peuplement en France. La Tour-d'Aigue, Ed. de l'Aube, $220 \mathrm{p}$.

Lévy J., 2003. Territoire. In Lévy J. et Lussault M., Dictionnaire de la Géographie et de l'espace des sociétés, Paris, Belin, 2003, p 907-919.

Ma Mung E., 2006. Négociations identitaires marchandes. Revue Européenne des Migrations Internationales, vol. 22, $\mathrm{n}^{\circ}$ 2, p. 83-93.

Panerai P., Croissances, 2005. Eléments d'Analyse Urbaine. Marseille, Parenthèses, 189 p.

Pervititch J., 2001. Sigorta Haritalarında Istanbul. Istanbul, AXA-OYAK, 338 p.

Piolle X., 1990. Mobilité, identités, territoires. Revue de Géographie de Lyon, vol. 65, n 3, p. 149-154.

Raffestin C., 1986. Territorialité : concept ou paradigme de la géographie sociale ? Geographica Helvetica, $\mathrm{n}^{\circ}$ 2, p. 91-96.

Roncayolo M., 1990. La ville et ses territoires. Paris, Folio Essais, Gallimard, 278 p.

SeraïdariK., 2011. Enjeux locaux autour d'un rituel à Sifnos : de l'analyse structurale à l'« objet patrimonial ». Balkanologie, vol. XII, n² 2 .

Sönmez S., 2008. Process of Electrification in Istanbul and its Modernization Process by the turn of Nineteeth Century: Tatavla as a case study. Mémoire de Master à l'Université de Bilgi, Faculté de Sciences et Lettres, Istanbul, $53 \mathrm{p}$.

Stolpe C., 1866. Plan de Constantinople avec ses faubourgs. Berlin, Institut d'Archéologie allemand, Istanbul.

Tarrius A., 2000. Les nouveaux cosmopolitismes, mobilités, identités, territoires. La Tour-d'Aigue, Ed. de l'Aube, $266 \mathrm{p}$.

Tsitsélikis K., 2009. Ta vakoufia ton ellivorthodokson koinotiton tis tourkias : nomikes opseis evos politikou problimatos. In Sinantisi sti poli to paron kai to mellon, Sindesmos Apofoiton Zografeiou, Athènes, KEMO, p. 153-188.

Valasiadis Y., 2005. Tatavla'da kar yağıyor. Istanbul, Dünya Kitapları, 110 p.

\section{NOTES}

1. Istanbul est composé d'immenses entités administratives, les belediye (municipalité) qui comportent plusieurs mahalles. Le mahalle étant la plus petite cellule représentante de l'Etat. Plus petit qu'un quartier, le mahalle est un espace de proximité immédiat. Entre les deux, il y a le semt. Kurtuluş, Feriköy, Pangaltı sont des semt. Le semt n'a pas d'existence administrative officielle, mais a néanmoins une existence sociale forte. Kurtuluş est donc un embrassement de plusieurs mahalles. Il pose ainsi un premier problème de délimitation spatiale propre à tous les quartiers d'Istanbul.

2. Le terme rum signifie historiquement les habitants de l'empire romain d'Orient. Il désigne l'ensemble de la population grécophone et orthodoxe d'Istanbul. Il a été et fait encore l'objet 
d'une idéologie nationaliste qui tend à assimiler un rum à un Grec. Aujourd'hui, la communauté rum recouvre une réalité et une diversité politiques et sociales bien plus complexes.

3. Toutes les rues du quartier de Tatavla, rebaptisé Kurtuluş, ont été renommées dans les années 1920. Je ne reviendrai pas sur ces changements toponymiques qui figureront dans ma thèse mais j'insiste sur le fait que ces nouvelles nominations sont des signes (voir introduction) à prendre en compte dans le mécanisme de production territoriale.

4. Suite aux accords de 1930 entre Vénizelos et Atatürk, de nombreux ressortissants grecs retournent s'installer à Istanbul.

5. Sinantisi sti poli to paron kai to mellon, (rencontre à Istanbul, le passé et le présent) Sindesmos Apofoiton Zografeiou, Athènes, KEMO, 2009.

6. Le projet My Istanbul lancé par Ömer Asan dans le cadre du programme Istanbul 2010 se compose de 80 récits de quartiers portant sur 80 quartiers différents.

7. Voir le groupe Facebook sur internet http://www.facebook.com/login/setashome.php? ref=home\#!/home.php?sk=group_166429960071912

\section{RÉSUMÉS}

Le quartier de Kurtuluş à Istanbul, héritier de l'ancien village de Tatavla, se forme et se développe jusqu'au milieu du XX $X^{\mathrm{e}}$ siècle. A partir des années 1950, le départ des minoritaires rum, jusque là quasi majoritaires, et l'arrivée des migrants anatoliens provoquent des ruptures démographiques considérables. Ces mobilités suscitent une diversité de modes territoriaux et produisent de nouvelles centralités, observables aussi par le bais du commerce et récemment à travers les stratégies mémorielles qui réédifient le passé rum du quartier. Enfin, ces territorialités participent à la recomposition du quartier et seront analysées à l'échelle d'une avenue phare, l'avenue de Kurtuluş.

The neighborhood of Kurtuluş, heritage of the ancient village of Tatavla, was formed and developed till the mid of the $20^{\text {th }}$ century. From 1950, along with the departure of the Rum minorities, which were considered till that time as quasi majorities, and with the arrival of Anatolian migrants, considerable demographical ruptures were provoked. Thoses mobilities create a diversity of territorial modes and give birth to new centralities, seen also throughout the commerce and recently from within the memorial strategies which rebuild the rum past of the neighborhood. Finally, those territorialities participate to the recomposition of the neigborhood and will be analysed through the scale of a headlight avenue, the avenue of Kurtuluş.

\section{INDEX}

Mots-clés : centralité, mémoire urbaine, mobilité, mutation des commerces, territorialité

Keywords : centrality, commercial mutation, mobility, territoriality, urban memory

Thèmes : Sur le Champ - Sur le Terrain 


\section{AUTEUR}

\section{CILIA MARTIN}

Cilia Martin, martin_cilia@yahoo.fr, est doctorante, CETOBAC/EHESS, sous la direction de Nathalie Clayer et boursière BAM de l'Institut Français d'Etudes Anatoliennes. Sa thèse porte sur : Kurtuluş sous l'effet des mobilités urbaines : formation et recomposition territoriale d'un quartier minoritaire d'Istanbul. 